\title{
Y... ¿DÓNDE QUEDAN LOS NIÑOS?... LA OTRA CARA DEL FEMINICIDIO
}

\author{
AND ... WHERE ARE THE CHILDREN? ... THE OTHER ASPECT OF \\ FEMICIDE
}

\author{
Percy Huaroc Llaja ${ }^{1}$
}

\section{RESUMEN:}

Breve análisis y debate sobre el artículo $108-B^{\circ}$ en nuestro Código Penal, en el cual se tipifica el delito especial de Feminicidio en su forma base y agravada, a su vez la discriminación positiva que se genera a partir de este tipo penal especial hacia un sector igualmente vulnerable, como es el caso de los niños, el cual merece en igual proporción una protección por parte del Estado Peruano.

Palabras Clave: Feminicidio, niños, protección.

\begin{abstract}
:
Brief analysis and debate on Article 108 - B of the Peruvian Criminal Code, which establishes the basic and aggravated criminal forms of Femicide. In turn, the paper discusses the positive discrimination resulting from this offense regarding an equally vulnerable group, as are the children, who deserve equal protection by the Peruvian government.
\end{abstract}

Key Words: Femicide. Feminicide. Children. Protection.

La Ley del Feminicidio promulgada en el año 2011 procuró aminorar la violencia de género producida en nuestro país tipificándola en un inicio dentro del tipo de parricidio ${ }^{2}$, al transcurrir de los años ésta violencia proclamada en la sociedad ante las mujeres se acrecentó de manera insólita, haciéndonos ver cada día vía prensa escrita, audio visual y radial las

\footnotetext{
1 Abogado por la Universidad Nacional Mayor de San Marcos, Master en Ciencias Penales por la misma Casa de Estudios. Jefe del Área Penal del Estudio Torres y Torres Lara Asociados Abogados. Profesor de Derecho Penal III en la Universidad Femenina Sagrado Corazón (UNIFE). Autor de diversos artículos publicados en "El Peruano" y revistas de Derecho. Fecha de recepción: 15 de setiembre de 2013

Fecha de aceptación: 07 de octubre de 2013

${ }^{2}$ Artículo $107^{\circ}$. Parricidio/Feminicidio

Si la víctima del delito descrito es o ha sido la cónyuge o la conviviente del autor, o estuvo ligada a él por una relación análoga el delito tendrá el nombre de feminicidio.
} 
barbaridades cometidas hacia las féminas; en consecuencia a ello, se promulgó el tipo penal especial de feminicidio, independiente del parricidio, ésta se dio mediante la incorporación del artículo $108-\mathrm{B}^{\mathrm{o} 3}$, publicada el 30 junio 2013.

$<$

Frente a lo señalado líneas más arriba, es necesario precisar cuál es el nacimiento del feminicidio y por qué es que nace esta violencia; además, de un análisis crítico de la norma emitida y la desprotección que se brinda a los menores de edad varones, ya que éstos también son sujetos de vulnerabilidad igual que las féminas menores y adolescentes; ¿es acaso una excesiva protección a las mujeres? ¿o, es dónde el Estado deja de velar por otra colectividad sujeta a violencia como es el caso de todos los menores de edad (incluido los niños), y no sólo sectores beneficiados?

En primer lugar, debemos empezar definiendo al feminicidio como: crimen de odio contra las mujeres; como el conjunto de formas de violencia que, en ocasiones, concluyen en asesinatos e incluso en suicidios; ésta afirmación nace a partir del trabajo de Diana Russell y Jill Radford, expuesto en su texto Femicide. The politics of woman killing (1992). ${ }^{4}$ Claro está que éste estudio denota la violencia y crueldad de cómo cientos de mujeres sufren y sufrieron agresiones indiscriminadamente llevándolas a la muerte por parte de su agresor.

${ }^{3}$ Artículo $108^{\circ}$-B.-Feminicidio

Será reprimido con pena privativa de libertad no menor de quince años el que mata a una mujer por su condición de tal, en cualquiera de los siguientes contextos:

1. Violencia familiar;

2. Coacción, hostigamiento o acoso sexual;

3. Abuso de poder, confianza o de cualquier otra posición o relación que le confiera autoridad al agente;

4. Cualquier forma de discriminación contra la mujer, independientemente de que exista o haya existido una relación conyugal o de convivencia con el agente.

La pena privativa de libertad será no menor de veinticinco años, cuando concurra cualquiera de las siguientes circunstancias agravantes:

\section{Si la victima era menor de edad;}

2. Si la víctima se encontraba en estado de gestación;

3. Si la víctima se encontraba bajo cuidado o responsabilidad del agente;

4. Si la víctima fue sometida previamente a violación sexual o actos de mutilación;

5. Si al momento de cometerse el delito, la víctima padeciera cualquier tipo de discapacidad;

6. Si la victima fue sometida para fines de trata de personas;

7. Cuando hubiera concurrido cualquiera de las circunstancias agravantes establecidas en el artículo 108.

La pena será de cadena perpetua cuando concurran dos o más circunstancias agravantes.

4 Extracto del peritaje rendido por Marcela Lagarde en el caso González y otras vs. México, conocido como "Campo Algodonero", resuelto por la Corte Interamericana de Derechos Humanos en su sentencia de noviembre de 2009. Disponible en: Sentencia de la Corte Interamericana de Derechos Humanos y Peritaje del Campo Algodonero vs. México. 
El feminicidio posee un carácter innovativo respecto a las legislaciones de América Latina debido a que surge de una necesidad de protección a las mujeres ya que a través de medios de prensa y la Policía Nacional se registran gran cantidad de asesinatos de éste tipo, tanto así que, en los últimos cuatro años, 547 mujeres fueron víctimas de feminicidio en el Perú de acuerdo a la información emitida por el Observatorio de la Criminalidad del Ministerio Público. ${ }^{5}$

Frente a estos sucesos, el Ministerio de la Mujer emitió una Resolución Ministerial, la cual define al feminicidio entendiendo a éste como el homicidio de mujeres cometido presuntamente por:

- La pareja o ex pareja de la víctima.

- Cualquiera de las personas comprendidas en la Ley de Protección frente a la Violencia Familiar.

- Alguna persona desconocida por la víctima siempre que el homicidio revele discriminación contra la mujer. ${ }^{6}$

Desde un punto de vista objetivo debe considerarse al feminicidio como una discriminación positiva ya que se emite normas de carácter especial para su protección al considerarse a las féminas como un grupo social con mayor vulnerabiliadad, por lo cuál el Estado debe buscar mejorar su calidad de vida. $^{7}$

A través de éste contexto, debemos señalar que de acuerdo al artículo 108- $\mathrm{B}^{\circ}$ sólo las mujeres sean niñas, adolescentes o adultas se consideran como las únicas agredidas en este tipo penal; sin embargo, los niños también son víctimas en algunos incisos descritos en el artículo 108- ${ }^{\circ}$. En consecuencia, podemos decir que da una desprotección a la población más frágil de toda la sociedad: entre ellos, los niños.

\footnotetext{
5 Diario Perú21, edición del domingo 04 de agosto del 2013. Disponible en: http://peru21.pe/actualidad/mas-60mujeres-murieron-victimas-feminicidio-peru-2143167

${ }^{6}$ De acuerdo a la Resolución Ministerial 110-2009-MIMDES que crea el Registro de Feminicidio y Tentativas.

${ }^{7}$ ¿Por qué lollaman "discriminación positiva"?, Pablo Molina. Disponible en:

http://www.ilustracionliberal.com/31/ por-que-lo-llaman-discriminacion-positiva-pablo-molina.html
} 
Tenemos que "Alrededor de 8 mil niños, niñas y adolescentes del Perú sufrieron maltrato psicológico y otros 4 mil maltrato físico durante el 2012, según reportes de los Módulos de Atención al Maltrato Infantil y del Adolescente en Salud (MAMIS) instalados en hospitales públicos." ${ }^{8}$

Estas cifras alarmantes engloban el maltrato psicológico y físico en menores de edad sin distinción de sexo alguno; en consecuencia, se tiene que el artículo sobre el feminicido excluye ciertas situaciones en las cuales las mujeres no son las únicas violentadas, concretamente hablamos de los numerales 1,2 y 3 del primer párrafo:

1. Violencia familiar;

2. Coacción, hostigamiento o acoso sexual;

3. Abuso de poder, confianza o de cualquier otra posición o relación que le confiera autoridad al agente;

... y en el siguiente párrafo, como agravante, los numerales $1,3,4,5,6$ y 7 :

1. Si la víctima era menor de edad;

3. Si la víctima se encontraba bajo cuidado o responsabilidad del agente;

4. Si la víctima fue sometida previamente a violación sexual o actos de mutilación;

5. Si al momento de cometerse el delito, la víctima padeciera cualquier tipo de discapacidad;

6. Si la víctima fue sometida para fines de trata de personas;

7. Cuando hubiera concurrido cualquiera de las circunstancias agravantes establecidas en el artículo 108.

8 Doce mil menores fueron víctimas de maltrato durante 2012. Disponible en: http://www.rpp.com.pe/2013-06-01doce-mil-menores-fueron-victimas-de-maltrato-durante-2012-noticia_600247.html 
Frente a los incisos citados debemos precisar que las estadísticas brindadas por el Instituto Nacional de Salud del Niño, del periodo que comprende entre los años 2006 - 2011 (Tabla 1), muestran las variables por las cuales los menores sufren diferentes formas de violencia.

Tabla 1. Caracterización del maltrato infantil en el Instituto Nacional de salud del Niño, Perú 2006-2011

\begin{tabular}{|c|c|c|}
\hline VARIABLE & $\mathrm{N}^{\circ}$ & $(\%)$ \\
\hline \multicolumn{3}{|l|}{ Edad del niño } \\
\hline Lactante & 242 & 13,5 \\
\hline Preescolar & 320 & 17,8 \\
\hline Escolar & 519 & 28.9 \\
\hline Adolescente & 717 & 39,9 \\
\hline \multicolumn{3}{|l|}{ Sexo del niño } \\
\hline Niño & 650 & 36,2 \\
\hline Niña & 1148 & 63.8 \\
\hline \multicolumn{3}{|l|}{ Sexo del agresor } \\
\hline Hombre & 1089 & 60.6 \\
\hline Mujer & 709 & 39,4 \\
\hline \multicolumn{3}{|c|}{ Parentesco del agres or con el agre dido } \\
\hline Madre o padre & 847 & 47,1 \\
\hline Otros familiares y vecinos & 827 & 46,0 \\
\hline Desconocido & 124 & 6,9 \\
\hline \multicolumn{3}{|c|}{ Grado de Instrucción del agres or } \\
\hline lletrada & 92 & 5,1 \\
\hline Pimaria completa & 172 & 9,6 \\
\hline Secundaria incompleta & 142 & 7,9 \\
\hline Secundaria completa & 1231 & 68,4 \\
\hline Superior incompleta & 9 & 0,5 \\
\hline Superior completa & 143 & 8.0 \\
\hline Técnica completa & 9 & 0,5 \\
\hline 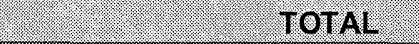 & 1798 & 100 \\
\hline
\end{tabular}

Aunque el mayor número de agraviadas sean las niñas, existe un $36,2 \%$ de niños que se encuentran excluidos de la protección del artículo que comentamos, y sus agresores no tendrían una sanción severa y ejemplar si en el caso sus víctimas fuesen niñas.

Esta investigación, realizada por los Módulos de Atención al Maltrato Infantil y del Adolescente en Salud, señala que los casos reportados poseen un expediente y un seguimiento para las víctimas y sus agresores. 
En la segunda tabla se puede visualizar los tipos de agresiones en los cuales también se encuentra niños conjuntamente con niñas $y$, de llevar estas agresiones a la muerte, también estarían incluidos los niños; sin embargo, no se encuentran dentro del tipo penal del artículo $108^{\circ}$-B.

\begin{tabular}{|c|c|c|c|c|c|c|c|c|}
\hline \multirow{3}{*}{$\begin{array}{l}\text { Tabla 2. Caracteristicas de } \\
\text { los tipos de agresión en el } \\
\text { INSN, Perú } 2006-2011\end{array}$} & \multicolumn{8}{|c|}{ Tipo de Agresión } \\
\hline & \multicolumn{2}{|c|}{ Sexual } & \multicolumn{2}{|c|}{ Abandono } & \multicolumn{2}{|c|}{ Psicologlico } & \multicolumn{2}{|c|}{ Fisico } \\
\hline & $\mathrm{N}^{\circ}$ & $(\%)$ & $\mathbf{N}^{\circ}$ & $(\%)$ & $\mathrm{N}^{\circ}$ & $(\%)$ & $\mathbf{N}^{\circ}$ & $(\%)$ \\
\hline \multicolumn{9}{|l|}{ Sexo del agredido } \\
\hline Niño & 234 & 26.7 & 264 & 44.2 & 85 & 41.5 & 67 & 54.9 \\
\hline Niña & 640 & 73.2 & 333 & 55.8 & 120 & 585 & 55 & 45.1 \\
\hline \multicolumn{9}{|l|}{ Edad del agredido } \\
\hline Lactante: & 40 & 46 & 179 & 30.0 & 13 & 63 & 10 & 82 \\
\hline Preescolar & 167 & 19.1 & 111 & 18.6 & 25 & 12.2 & 17 & 13.9 \\
\hline Escolar & 279 & 31.9 & 139 & 23.8 & 50 & 14.4 & 51. & 41.8 \\
\hline Adolescente & 388 & 44.4 & 168 & 28.1 & 117 & 57.1 & 44 & 36.1 \\
\hline \multicolumn{9}{|l|}{ Parentes co del agres or } \\
\hline Madreo o padre & 249 & 28.5 & 412 & 69.0 & 110 & 53.7 & 76 & 62.3 \\
\hline Otros familares y vecinos & 548 & 627 & 168 & 28.1 & 69 & 337 & 42 & 34.4 \\
\hline Desconocido & 77 & 8.8 & 17 & 2.9 & 26 & 12.7 & 4 & 3.3 \\
\hline TOTAL & 874 & 48.6 & 597 & 33.2 & 205 & 11.4 & 122 & 6.8 \\
\hline
\end{tabular}

Como ya se ha mencionado, estos datos recién conocidos en el pasado mes de junio del 2013, son brindados por los Módulos de Atención al Maltrato Infantil y del Adolescente en Salud (MAMIS) ubicados en diferentes nosocomios del país.

Otros datos en forma general que se brindan respecto a agresiones a menores, es que, en dos meses del año 2012 (periodo Enero - Febrero) del 30\% de casos de violencia familiar, las víctimas eran del género masculino; claro está, todos menores de edad, comprendidos de 0 a 17 años.

\footnotetext{
9 Infografía, RPP Noticias. Casos de violencia familiar en el 2012. Disponible en: http:// www.rpp.com.pe/2012-11-22-casosde-violencia-familiar-en-el-2012-noticia__542895.html, Consultado el 5 de septiembre de 2013.
} 


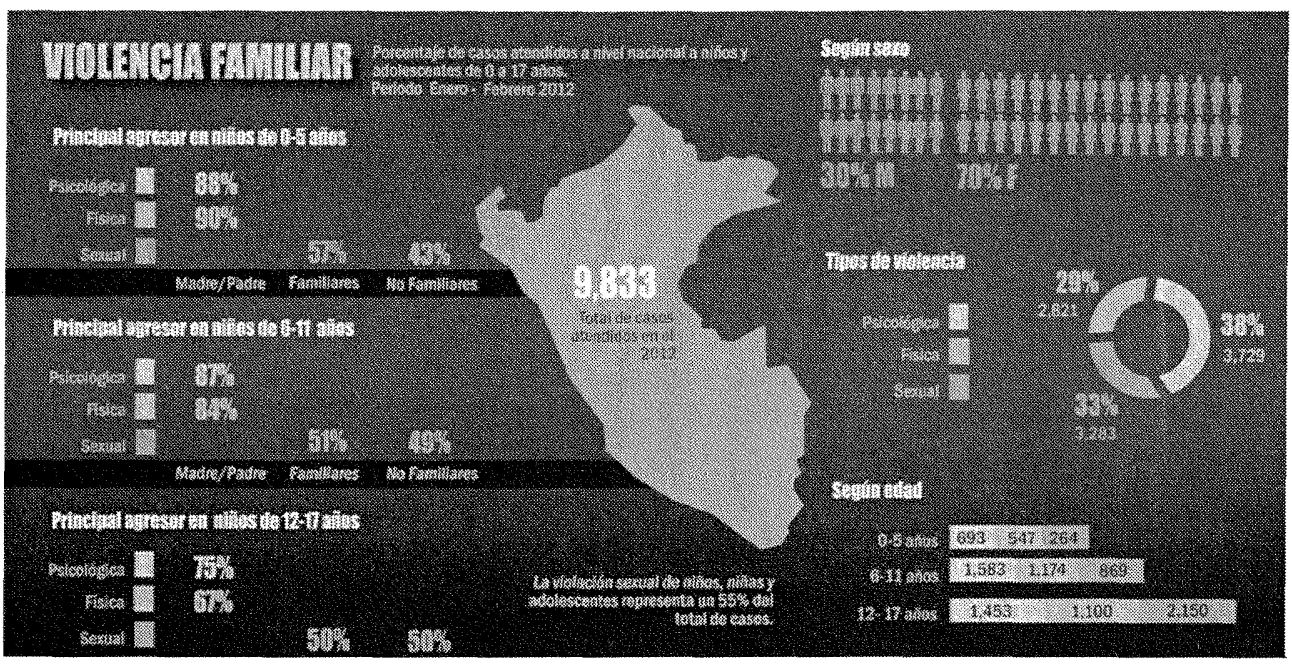

Un punto alarmante de esta situación es que, en cuadros estadísticos actuales, el Ministerio de la Mujer y Poblaciones Vulnerables, en sus avances al 2013, no hace ninguna referencia sobre cifras donde el agraviado pueda ser un varón menor de edad respecto a un homicidio, solo señala la presencia de varones en los casos atendidos de violencia familiar; en casos en el que como consecuencia de la violencia familiar ocurriese la muerte, no entrarían en estás estadísticas: ${ }^{10}$

Cuadro Nª: Casos de Feminicidio y/o tentativas, según grupo de edad de la víctima.

\begin{tabular}{|ccccc|}
\hline $\begin{array}{c}\text { Grupos de } \\
\text { Edad }\end{array}$ & Feminicidio & Tentativa & Total & $\%$ \\
\hline $0-5$ años & 1 & 0 & 1 & $0.7 \%$ \\
\hline $6-11$ años & 2 & 0 & 2 & $1.4 \%$ \\
\hline $12-17$ años & 4 & 5 & 9 & $6.4 \%$ \\
\hline $18-25$ años & 22 & 29 & 51 & $36.4 \%$ \\
\hline $26-35$ años & 19 & 30 & 49 & $35.0 \%$ \\
\hline $36-45$ años & 8 & 11 & 19 & $13.6 \%$ \\
$46-59$ años & 5 & 1 & 6 & $4.3 \%$ \\
\hline 60 a + años & 1 & 2 & 3 & $21 \%$ \\
\hline Sin dato & 0 & 0 & 0 & $0.0 \%$ \\
\hline Total & 62 & 78 & 140 & $100 \%$ \\
\hline
\end{tabular}

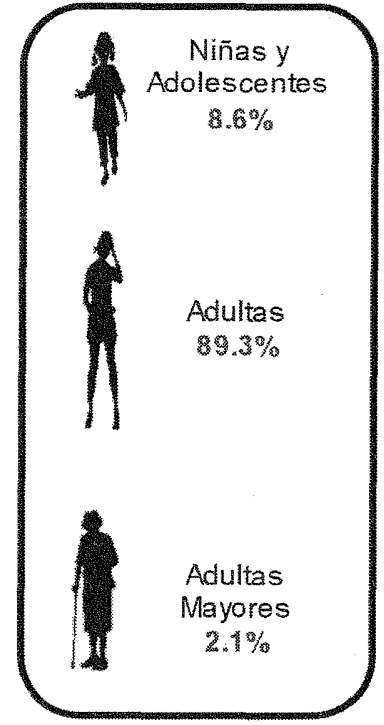

10 Resumen Estadístico de Casos de Feminicidio y Tentativa de feminicidio a Nivel Nacional. Período: Enero - Julio 2013 ( Preli m in a r). $\quad$ Disponible e $\mathrm{n}$ : http:/ / www.mimp.gob.pe/index.php?option $=$ com_content\&view =article\&tid=1401:boletines-y-resumenesestadisticos\&catid $=130$ 

4. El artículo $108^{\circ}-\mathrm{B}$ del Código Penal que regula y sanciona el Feminicidio, excluye a un gran sector de personas, en este caso niños víctimas de homicidio, por lo que se hace necesaria la modificación de dicho artículo, el mismo que debe incluir necesariamente a los menores de edad por ser una población vulnerable; de lo contrario, estaríamos discriminándolos.

\section{REFERENCIAS}

Caso Gónzales y otros ("Campo Algodonero") vs. México (Corte Interamericana de Derechos Humanos 16 de Noviembre de 2009).

La República.pe (28 de noviembre de 2011) Fatal: 104 niños fueron asesinados. En: Diario La República (on line). Lima, Perú. Disponible en Internet:http://www.larepublica.pe/28-11-2011/fatal-104-ninos-fueronasesinados. Consultado el 7 de setiembre de 2013.

Ministerio de la Mujer y Poblaciones Vulnerables. Resumen Estadístico de Casos de Feminicidio y Tentativa de Feminicidio a Nivel Nacional Período: Enero - Julio 2013 (Preliminar). Disponible en Internet:http://www.mimp.gob.pe/index.php?option=com_content\&view $=$ article \&id $=1401$ :boletines-y-resumenes-estadisticos $\&$ catid $=130$. Consultado el 7 de setiembre.

Molina, Pablo.( $\mathrm{N}^{\circ} 31$ Reseñas) ¿Por qué lo llaman "discriminación positiva"? En: La Ilustración Liberal Revista Española y latinoamericana (on line). España: La Ilustración Liberal Madrid. Disponible en Internet: http://www.ilustracionliberal.com/31/por-que-lo-llaman-discriminacionpositiva-pablo-molina.html. Consultado el 05 de setiembre de 2013.

Perú21.pe (4 de Agosto de 2013) Más de 500 casos de feminicidio se han registrado en el Perú. En Diario Perú21 edición online. Lima, Perú. Disponible en Internet: http://peru21.pe/actualidad/mas-60-mujeresmurieron-victimas-feminicidio-peru-2143167. Consultado el 28 de agosto de 2013.

Red de Investigadoras por la Vida y la Libertad de las Mujeres A.C. (2010). Sentencia de la Corte Interamericana de Derechos Humanos y Peritaje del Campo Algodonero vs. México. México: Milagros Agrupación Feminista, A.C. 
RPP Noticias (22 de noviembre del 2012) Casos de violencia familiar en el 2012. En: RPP Noticias (on line). Lima, Perú. Disponible en Internet: http://www.rpp.com.pe/2012-11-22-casos-de-violencia-familiar-en-el2012-noticia_542895.html. Consultado el 5 de septiembre de 2013.

RPP Noticias (1 de Junio de 2013) Doce mil menores fueron víctimas de maltrato durante 2012. En RPP Noticias (online). Lima, Perú. Disponible en Internet: http://www.rpp.com.pe/2013-06-01-doce-mil-menores-fueronvictimas-de-maltrato-durante-2012-noticia_600247.html. Consultado el 5 de septiembre de 2013.

Resolución Ministerial 110-2009-MIMDES, publicado el 09 de marzo de 2009 en el Diario Oficial “El Peruano". Lima. 